Original Article

\title{
Patterns of physical activity and exercise after lumbar surgery among Japanese patients with lumbar spinal stenosis
}

\author{
Daisuke Higuchi, RPT, PhD ${ }^{1 *}$, Yu Kondo, RPT ${ }^{2)}$, TAKahiro Miki, RPT, MSci²) \\ 1) Department of Physical Therapy, Takasaki University of Health and Welfare: 501 Nakaorui-machi, \\ Takasaki-shi, Gunma 370-0033, Japan \\ 2) Sapporo Maruyama Orthopedic Hospital, Japan
}

\begin{abstract}
Purpose] The purpose of this study was to assess the feasibility of classifying the patterns of physical activity and exercise after surgery for lumbar spinal stenosis in Japanese patients and describe the characteristics of the patient groups. [Participants and Methods] We evaluated Japanese patients diagnosed as having lumbar spinal stenosis and underwent surgery. The frequencies of the 15 types of physical activity and exercise recommended in Kenko Nippon 21 (Japanese policy for health promotion) were investigated by mail. The study included 102 respondents (median age, 69 years [range, 34-88 years]; 55 males and 47 females). A hierarchical cluster analysis was used for grouping according to the physical activity and exercise patterns. The Holm method and residual analysis were used for comparisons of the frequencies of the physical activity and exercise patterns and basic demographics among the groups. [Results] Three clusters, namely clusters A (younger), B (frail older), and C (active older), were identified from the dendrogram. The participants in cluster A frequently performed paid work. In cluster B, the frequencies of all the physical activity and exercise patterns were low. The older people in cluster C regularly performed stretching/light-intensity exercises, walking, muscle-strengthening exercises, and house and garden maintenance tasks. [Conclusion] We found that the physical activity and exercise after lumbar surgery in Japanese patients could be divided into three patterns.

Key words: Lumbar spinal stenosis, Health promotion, Physical activity
\end{abstract}

(This article was submitted Sep. 18, 2020, and was accepted Nov. 25, 2020)

\section{INTRODUCTION}

The population in Japan is aging, with the average life expectancy increasing while the birth rate is decreasing. Accordingly, the burden of cost of social security is increasing. To address this issue, the Japanese Ministry of Labor, Health, and Welfare formulated the Kenko Nippon 21 (Healthy Japan 21) in 2000 and mandated all 47 prefectures to promote health to increase health-related quality of life (HRQOL) and consequently decrease the social security cost. The first stage of the Kenko Nippon 21 was implemented from 2000-2012, and the second stage is in effect until 2022, with the following six dimensions set as targets: nutrition and dietary habits; physical activity (PA) and exercise (Ex); rest; alcohol drinking; tobacco smoking; dental and oral health. Unfortunately, many targets have not been accomplished, and thus, activities to achieve the targets of each dimension need to be promoted.

$\mathrm{PA} / \mathrm{Ex}$ is one of the prospective approaches to health promotion. PA is positively associated with sleep quality ${ }^{1,2)}$ and HRQOL among healthy older people ${ }^{3,4)}$. Further, PA/Ex intervention has been reported to have a positive effect on mobility and physical functioning among older people ( $\geq 65$ years) with physical impairments ${ }^{5}$ and frailty ${ }^{6}$. PA is also significantly correlated with HRQOL, including in those aged $<65$ years ${ }^{7}$. Moreover, PA is associated with various work-related factors

*Corresponding author. Daisuke Higuchi (E-mail: higuchi-d@takasaki-u.ac.jp)

(C2021 The Society of Physical Therapy Science. Published by IPEC Inc.

(c) $(-)$ This is an open-access article distributed under the terms of the Creative Commons Attribution Non-Commercial No Deriva-

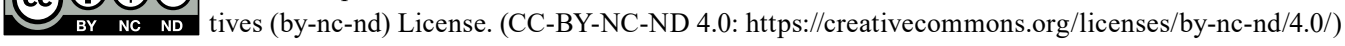


such as low work ability ${ }^{8)}$, perceived stress at work ${ }^{9}$, and receiving disability pension ${ }^{10)}$. A relationship between PA and back pain has also been established; workers with extremely high or low PA are more likely to complain of low back pain (LBP), while workers with moderate PA are the least likely to complain of $\mathrm{LBP}^{11)}$. These results collectively support that health promotion through PA/Ex should be performed in both individuals with and without morbidity.

LSS is one of the most common spinal disorders among the middle-aged (45-64 years) and older people, thus making individuals with LSS appropriate targets for health promotion through PA/Ex. Additionally, people with LSS have health and social problems despite undergoing lumbar surgery. In Japan, approximately 3.65 million people have LSS ${ }^{12)}$. The common symptoms of LSS are low back pain, leg pain and dysesthesia, and muscle weakness in the legs. When conservative treatment is not effective and the LSS impairs the performance of activities for daily living due to pain and/or dysesthesia in the low back and/or the legs, patients undergo surgical treatment for symptom management ${ }^{13)}$. However, pain and/or dysesthesia do not always completely disappear after surgery ${ }^{14}$. About $25 \%$ of postoperative LSS patients still report pain and/or dysesthesia and require medication ${ }^{15}$. Accordingly, individuals with LSS have poorer HRQOL than healthy people even after undergoing ${ }^{16)}$. They also have a less than $50 \%$ probability of returning to work after surgery ${ }^{17,18)}$.

The Kenko Nippon 21 describes the characteristics of each life stage and highlights the importance of health promotion conforming to the life stage. Therefore, the targets of PA/Ex are separated into two by age: 20-64 years (young to middle age adults) and 65 years or older (older adults). Young to middle age adults often spend a considerable time working during the daytime, while older adults after retirement can use daytime according to their preference. Moreover, physical activity can be more varied among older people.

We wanted to understand the patterns of PA/Ex among individuals with LSS to develop PA/EX interventions that improve physical and mental function as well as HRQOL after lumbar surgery in these patients, particularly those who have chronic pain and/or dysesthesia. We hypothesized that the patterns of PA/Ex after surgery for LSS differ between Japanese people aged 20 years or older and should thus be separated according to the patient's life stage and style. In the current study, we aimed to assess the feasibility of classifying Japanese people according to the pattern of PA/Ex after surgery for LSS and, if the classification is successful, describe the characteristics of the different groups based on our classification.

\section{PARTICIPANTS AND METHODS}

This cross-sectional study was approved by the ethical committee of Sapporo Maruyama Orthopedic Hospital (approval number: 000025). The participants were individuals who were diagnosed with LSS and underwent surgical treatment between September 2017 and August 2018 in Sapporo Maruyama Orthopedic Hospital in Japan. Given that the pathology of LSS involves spinal and nerve root canal stenosis through intervertebral disc herniation, hypertrophy of the ligamentum flavum, hypertrophy of the facet joint, spondylolisthesis, osteophyte, and ectopic fat tissue, lumbar disc herniation were considered as subtypes of LSS in this study. Individuals aged less than 20 years and those whose current addresses were unknown were excluded.

We developed a set of questionnaires comprising three sections on demographic data, HRQOL using EuroQOL 5 dimensions 5-level (EQ-5D-5L), and 15 types of PA/Ex introduced in the Kenko Nippon 21 (See below for details). To combine the response data with the medical records of Sapporo Maruyama Orthopedic Hospital, respondents were asked to provide his/her name. After the questionnaire was developed, the addresses of people who met the inclusion criteria were extracted from the Sapporo Maruyama Orthopedic Hospital database and were distributed by mail in November 2019. Explanatory leaflets about this study, consent forms to participate in this study, and return envelopes were also enclosed. If they agreed to participate in the study, they were asked to return the answered questionnaire and the consent to participate in the study form to Sapporo Maruyama Orthopedic Hospital within 2 weeks of arrival. The returned questionnaires were tabulated by the authors and converted into electronic data.

Patient age was determined using the questionnaire, while data on gender, date of lumbar surgery, and operative methods were extracted from the medical records. The average intensities of LBP and pain and numbness of the legs for the previous month were assessed using the 11-point numerical rating scale (NRS), with 0 points defined as "no pain or numbness" and 10 points as "unbearable pain or numbness". The NRS has been previously verified to be a reliable and valid scale for assessing pain intensity ${ }^{19)}$.

EQ-5D-5L was used to assess HRQOL. The EQ-5D-5L consists of five questions concerning mobility, self-care, usual activities, pain/discomfort, and anxiety/depression. Severities of these five dimensions are divided into five levels ${ }^{20)}$. The construct validity of the EQ-5D-5L has been confirmed in patients with stroke $\mathrm{e}^{21)}$ and osteoarthritis ${ }^{22)}$. The EQ-5D-5L index scores, which were calculated by replies to the five questions, ranges from 0 to 1 , with 0 - and 1- points representing death and full healthy status, respectively ${ }^{23)}$.

We evaluated the following 15 types of PA/Ex introduced in the Kenko Nippon 21: (1) paid works; (2) activities in the community and volunteers; (3) intellectual and cultural activities such as music, and art appreciation and hobbies; (4) stretching and light-intensity exercises; (5) walking; (6) muscle-strengthening exercises of the trunk and legs; (7) hiking, picnic, orienteering, and camping; (8) traveling and hosteling; (9) dancing (e.g., social dances, folk dances, classical Japanese dances, yoga and tai chi); (10) maintenance tasks of the house and garden including kitchen garden; (11) golf including ground golf, mini golf, and target-bird-golf; (12) gateball (Japanese croquet), bowling, and lawn bowling; (13) lawn and 
table tennis; (14) billiards, quoits, darts, and frisbee; and (15) swimming, walking, dances, and underwater exercises. The frequencies of each PA/Ex for the previous month were investigated using the 5-point Likert scale (1: never; 2: irregular; 3: 1-2 days per week; 4: 3-4 days per week; 5: 5 days or more per week).

First, missing values were complemented using the multiple imputation method by chained equation. We set the number of substitutions at 10. Second, respondents were classified based on frequencies of PA/Ex using the hierarchical cluster (CL) analysis (dissimilarity index: the Euclidean distance; clustering method: the furthest neighbor method), and we assessed whether respondents could be categorized into three CLs using the dendrogram. If, as hypothesized, the dendrogram could classify the respondents into three clusters, the descriptive statistics values of scores of the assessed items were calculated in each group, and the scores were compared among the three groups using the Holm method for the ordinal and ratio variables and the residual analysis for the categorical variables.

The multiple imputation method was performed using R version 3.6 (Foundation for Statistical Computing, Vienna, Austria), while other statistical analyses were performed using HAD version $16.1^{24}$ ). A probability level of 0.05 was considered statistically significant.

\section{RESULTS}

Questionnaires were sent to 176 individuals who underwent lumbar surgery more than 1 year earlier in Sapporo Maruyama Orthopedic Hospital, and the response rate was $59 \%(\mathrm{n}=102)$. Three sets of questionnaires were returned because of unknown address. The median participant age was 69 years [range, 34-88 years], and 55 and 47 were male and female, respectively.

In total, $8 / 102$ participants $(7.8 \%)$ retuned questionnaires with missing responses. The total percentage of missng values in all 23 questions was $0.6 \%(15 / 2346)$. These missing values were complemented using the multiple imputation method, and the absolute data of 102 respondents were used for the following analyses.

A dendrogram categorizing 102 participants into 25 clusters (CLs1-25) based on the frequency scores of the 15 types of PA/Ex is shown in Fig. 1. The dendrogram indicated three CLs according to the cut-off line. CLs 1 to 9, 10 to 17, and 18 to 25 were named as CLs A, B, and C, respectively. CLs A, B, and C comprised 40, 51, and 11 participants, respectively. The participant characteristics by CL are shown in Table 1. Participants in CL A were significantly younger (percentage of participants aged 65 years or older: 32.5\% [13/40]) than those in CLs B (76.5\% [39/51]) and C (81.8\% [9/11]). CL A also included more men, had lower number of posterior interbody fusion, and higher EQ-5D-5L. Meanwhile, CLs B and C included a higher number of female and lower number of laminoplasty, respectively. There were no significant differences in the interval between surgery and NRS of LBP and leg pain and numbness among the three CLs.

The frequencies of PA/Ex in CLs A, B, and C are summarized in Table 2. We found significantly higher frequencies of paid work in CL A compared with CL B and C $(p<0.01)$. There were significantly higher frequencies of intellectual and cultural activities in CL C versus CL B $(\mathrm{p}<0.05)$. There were also higher frequencies of stretching and light-intensity exercises, walking, and muscle-strengthening exercises in CL C than CL A and B $(\mathrm{p}<0.01)$. Moreover, maintenance tasks for the house and garden, including kitchen gardening were also higher in CL C than CL A and $B(p<0.05)$. There were no higher frequencies of PA/Ex in CL B compared with CL A and C.

\section{DISCUSSION}

The first purpose of this study was to assess whether Japanese people could be classified based on the pattern of PA/Ex after surgery for LSS. Through the hierarchical CL analysis, we identified three groups (CLs A, B, and C). This classification met our hypothesis and supported the validity of making targets of PA/Ex at each life stage stipulated by the Kenko Nippon 21 .

Behavioral characteristics of CL A included higher frequency of paid work compared with the other two CLs. Meanwhile, CL C showed higher frequencies of intellectual and cultural activities; stretching and light-intensity exercises; walking; muscle-strengthening exercises; and maintenance tasks of house and garden, including gardening. Unlike CLs A and C, the frequencies of all PA/Ex were low in CL B. These PA/Ex can be performed inside the house; thus, we considered stretching and light-intensity exercises, walking, muscle-strengthening exercises, and maintenance tasks of the house and garden as potential components of PA/Ex intervention programs. In contrast, sports and recreational activities, which needed more money and time, specific facilities, or colleagues, were not regularly performed in all CLs.

Many people in CL A (work-centered group) were aged less than 65 years, men, and had a higher HRQOL than the other CLs. The employment of individuals aged less than 65 years is legally protected, and government data show that the number of male full-time workers is more than 1.5 times higher than that of female workers in Japan. The EQ-5D-5L user guide shows that the EQ-5D-5L index scores of younger people are greater than those of older people. Moreover, it was reported that returning to working would positively influence the HRQOL ${ }^{25)}$, and that HRQOL and psychological distress in working people with coronary heart disease were better than in non-working people ${ }^{26}$.

Participants in CLs B (non-active group) and C (active group) were mainly aged over 65 years. Although both CLs B and $\mathrm{C}$ were coetaneous, CL B included more females, while CL C had a lower rate of laminoplasty. The amount of PA based on the triaxial accelerometer in older Japanese females was found to be significantly lower than that of older men ${ }^{27)}$, and this 


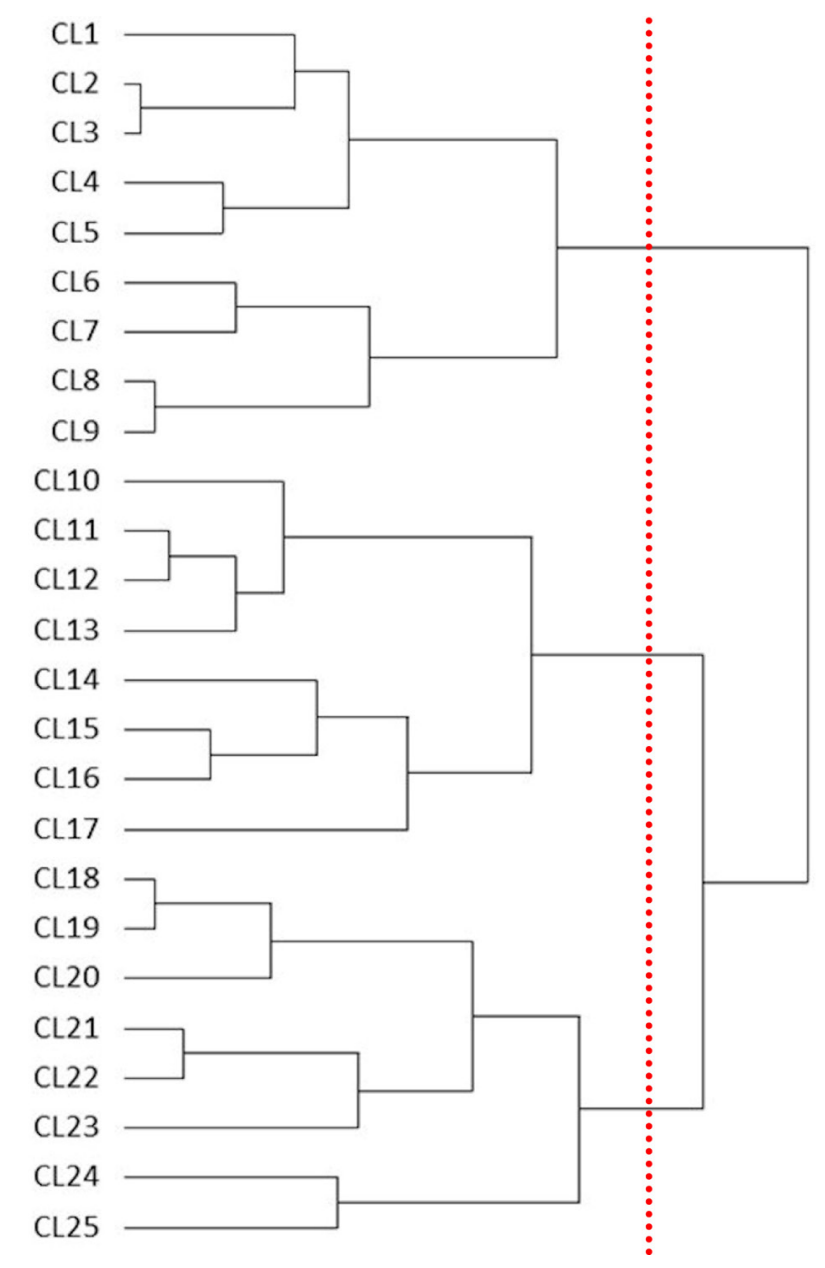

Fig. 1. Dendrogram clustering the 102 responders based on patterns of physical activities and exercises.

This dendrogram was drawn after placing 102 responders into 25 clusters (CL 1-25). The dotted line is the cut-off line; three clusters were made: CL A, CLs 1-9; CL B, CLs 10-17; and CL C, CLs 18-25.

may have caused the higher number of older females in CL B in this study. The intensities of low back pain and leg pain/ numbness did not significantly differ between the CLs B and C, but no participant in CL C has undergone laminoplasty to decompress the nerve root. This finding suggested that radiological findings of LSS in the CL C were more severe than those in CL B because decompression techniques such as laminoplasty and discectomy were frequently used in CL C, while instrumentation techniques, i.e., posterior interbody fusion, were frequently used in CL B. Although, the EQ-5D-5L index scores did not significantly differ between CLs B and C, we believe that this finding does not necessarily indicate that there was no association between PA/Ex and HRQOL. It was difficult to assess the relationship between PA/Ex and HRQOL by comparing only HRQOL between CLs B and C. CL B involved not only participants whose HRQOL was low due to low PA/ Ex, but also those who did not want to perform PA/Ex because they had inadequate physical ability. Meanwhile, the CL C included not only people who succeeded in maintaining high HRQOL by doing regular PA/Ex, but also those who had yet to benefit from PA/Ex because they only started the interventions/activity.

The classification of PA/Ex after lumbar surgery in this study indicates that PA/Ex interventions should be individualized and modified according to the three patterns of PA/Ex: 1) more frequent paid work, 2) less frequent PA/Ex, and 3) more frequent daily PA such as walking, stretching/light exercise, and household tasks. First, workers (CL A) spend a high amount of time performing work-related tasks, and thus it is difficult to allow time for sports and recreation. Therefore, measures to increase the amount of PA should be provided at the work site ${ }^{28,29)}$ considering the job type ${ }^{30)}$. Second, older people with low frequency of PA/Ex (CL B), particularly females, are at risk of frailty ${ }^{31)}$. Appropriate PA/Ex by the older population, namely, stretching and light-intensity exercises, walking, muscle-strengthening exercises, maintenance tasks of house, and garden including kitchen gardening, at low frequencies and intensities are recommended as the first step. Combinations with cognitive interventions to motivate PA/Ex are promising ${ }^{32)}$. Third, for older people with high frequency of PA/Ex (CL $\mathrm{C})$, the recommendation is to increase intensity or time rather than frequency of common PA/Ex or introducing sports and 
Table 1. Baseline clinicodemographic characteristics of the participants by cluster of physical activity and exercise

\begin{tabular}{|c|c|c|c|}
\hline & $\begin{array}{c}\text { Cluster A } \\
(\mathrm{n}=40)\end{array}$ & $\begin{array}{c}\text { Cluster B } \\
(\mathrm{n}=51)\end{array}$ & $\begin{array}{c}\text { Cluster C } \\
(\mathrm{n}=11)\end{array}$ \\
\hline Age $(\text { years })^{\dagger}$ & $59 \pm 8.3[34-86]^{\mathrm{a}}$ & $72 \pm 6.0[40-88]$ & $74 \pm 3.3[45-82]$ \\
\hline Gender (male; female) & $30^{\mathrm{c}} ; 10^{\mathrm{d}}$ & $19^{\mathrm{d}} ; 32^{\mathrm{c}}$ & $6 ; 5$ \\
\hline Days after surgery $^{\dagger}$ & $572 \pm 83.9$ [396-759] & $515 \pm 98.8[382-779]$ & $552 \pm 108.0[410-773]$ \\
\hline \multicolumn{4}{|l|}{ Operative methods } \\
\hline Laminoplasty & $14(35.0 \%)$ & $12(23.5 \%)$ & $0(0 \%)^{\mathrm{e}}$ \\
\hline Posterior interbody fusion & $15(37.5 \%)^{\mathrm{d}}$ & $33(64.7 \%)$ & $9(81.8 \%)$ \\
\hline Discectomy & $11(27.5 \%)$ & $6(11.8 \%)$ & $2(18.2 \%)$ \\
\hline NRS for low back pain ${ }^{\dagger}$ & $0.5 \pm 1.1[0-5]$ & $2 \pm 1.5[0-8]$ & $2 \pm 1.8[0-6]$ \\
\hline NRS for leg pain ${ }^{\dagger}$ & $0 \pm 1.0[0-5]$ & $1 \pm 1.5[0-7]$ & $2 \pm 1.5[0-7]$ \\
\hline NRS for leg numbness ${ }^{\dagger}$ & $0 \pm 1.0[0-7]$ & $1 \pm 1.5[0-8]$ & $3 \pm 2.3[0-8]$ \\
\hline EQ-5D-5L ${ }^{\dagger}$ & $0.81 \pm 0.14^{\mathrm{b}}[0.48-1.00]$ & $0.76 \pm 0.08[0.01-1.00]$ & $0.70 \pm 0.08[0.51-1.00]$ \\
\hline
\end{tabular}

†Values are presented as mean (median) \pm (quartile deviation) [range].

$*$ Anterior or posteriolateral fusions were combined as needed.

${ }^{\mathrm{a}} \mathrm{p}<0.01$ (vs. cluster $\mathrm{B}$ and $\mathrm{C}$ ); ${ }^{\mathrm{b}} \mathrm{p}<0.05$ (vs. clusters $\mathrm{B}$ and $\mathrm{C}$ ); ${ }^{\mathrm{c}}$ significantly higher values from the adjusted standardized residuals $(\mathrm{p}<0.01)$; ${ }^{\mathrm{d}}$ significantly lower values from the adjusted standardized residuals $(\mathrm{p}<0.01)$; ${ }^{\mathrm{e}}$ significant lower values from the adjusted standardized residuals $(\mathrm{p}<0.05)$.

NRS: numerical rating scale; EQ-5D-5L: EuroQOL 5 dimensions 5-level.

Table 2. Frequencies of physical activities and exercises by cluster

\begin{tabular}{llll}
\hline & $\begin{array}{c}\text { Cluster A } \\
(\mathrm{n}=40)\end{array}$ & $\begin{array}{c}\text { Cluster B } \\
(\mathrm{n}=51)\end{array}$ & $\begin{array}{c}\text { Cluster C } \\
(\mathrm{n}=11)\end{array}$ \\
\hline Paid work & $5 \pm 0[1-5]^{\mathrm{a}}$ & $1 \pm 0[1-2]$ & $1 \pm 0[1-3]$ \\
Activities in the community and volunteers & $1 \pm 0[1-2]$ & $1 \pm 0[1-4]$ & $1 \pm 0.3[1-5]$ \\
Intellectual and cultural activities & $1 \pm 0.5[1-4]$ & $1 \pm 0.5[1-4]$ & $2 \pm 1.3[1-5]^{\mathrm{b}}$ \\
Stretching and light-intensity exercises & $2 \pm 1.1[1-5]$ & $2 \pm 1.0[1-5]$ & $4 \pm 0.5[2-5]^{\mathrm{c}}$ \\
Walking & $1 \pm 0.5[1-5]$ & $2 \pm 0.5[1-5]$ & $4 \pm 0.5[2-5]^{\mathrm{c}}$ \\
Muscle-strengthening exercises & $1 \pm 0.5[1-5]$ & $1 \pm 0.5[1-5]$ & $4 \pm 1.3[1-5]^{\mathrm{c}}$ \\
Hiking, picnic, orienteering, and camping & $1 \pm 0[1-2]$ & $1 \pm 0[1-3]$ & $1 \pm 0[1-1]$ \\
Dancing & $2 \pm 0.5[1-2]$ & $1 \pm 0.5[1-2]$ & $1 \pm 0.5[1-2]$ \\
Traveling and hosteling & $1 \pm 0[1-4]$ & $1 \pm 0[1-3]$ & $1 \pm 0[1-4]$ \\
Maintenance tasks of the house and garden & $1 \pm 0.5[1-5]$ & $2 \pm 0.5[1-5]$ & $4 \pm 1.8[1-5]^{\mathrm{d}}$ \\
Golf & $1 \pm 0[1-4]$ & $1 \pm 0[1-3]$ & $1 \pm 0[1-3]$ \\
Gateball, bowling, and lawn bowling & $1 \pm 0[1-2]$ & $1 \pm 0[1-3]$ & $1 \pm 0.3[1-3]$ \\
Lawn and table tennis & $1 \pm 0[1-3]$ & $1 \pm 0[1-2]$ & $1 \pm 0[1-1]$ \\
Billiards, quoits, darts, and frisbee & $1 \pm 0[1-1]$ & $1 \pm 0[1-1]$ & $1 \pm 0[1-1]$ \\
Swimming and underwater exercise & $1 \pm 0[1-3]$ & $1 \pm 0[1-4]$ & $1 \pm 0[1-4]$ \\
\hline
\end{tabular}

Values are presented as mean (median) \pm (quartile deviation) [range]. ${ }^{a} \mathrm{p}<0.01$ (vs. cluster $\mathrm{B}$ and $\mathrm{C}$ ); ${ }^{\mathrm{b}} \mathrm{p}<0.05$ (vs. cluster $\mathrm{B}$ ); ${ }^{c} \mathrm{p}<0.01$ (vs. cluster $\mathrm{A}$ and $\mathrm{B}$ ); ${ }^{\mathrm{d}} \mathrm{p}<0.05$ (vs. cluster $\mathrm{A}$ and $\mathrm{B}$ ).

recreational activities. Although it is necessary to consider the background of the people as well as their preferences and economic and cultural background, the classification of PA/Ex in this study would help develop individualized programs for health promotion through PA/Ex among participants with LSS who underwent surgery.

This study has two major limitations. First, it is impossible to clarify the relationship between PA/Ex and HRQOL because this was a cross-sectional study. The effect of PA/Ex on HRQOL in individuals with high PA/Ex and low HRQOL was not evaluated because of the low intensity or short duration of PA/Ex. We intend to examine the internal structure of both CLs B and $\mathrm{C}$ to understand further the association between PA/Ex and HRQOL among older people who underwent lumbar surgery. Second, this study was conducted from late autumn to early winter; seasons and climates ${ }^{33,34)}$ and area of residence (urban or rural $)^{35)}$ are known to influence the amount and patterns of PA/Ex. Against these limitations, longitudinal studies, including intervention studies and comparative studies at multiple sites in different climatic and cultural backgrounds, can deepen and reinforce the findings of this study. 


\section{Funding}

This research was funded by the Japan Society for the Promotion of Science under Grant (KAKENHI) [grant number JP19K11201].

\section{Conflict of interest}

There is no conflict of interest to be disclosed.

\section{REFERENCES}

1) Christie AD, Seery E, Kent JA: Physical activity, sleep quality, and self-reported fatigue across the adult lifespan. Exp Gerontol, 2016, 77: 7-11. [Medline] [CrossRef]

2) Gothe NP, Ehlers DK, Salerno EA, et al.: Physical activity, sleep and quality of life in older adults: influence of physical, mental and social well-being. Behav Sleep Med, 2020, 18: 797-808. [Medline] [CrossRef]

3) Halaweh H, Willen C, Grimby-Ekman A, et al.: Physical activity and health-related quality of life among community dwelling elderly. J Clin Med Res, 2015, 7: 845-852. [Medline] [CrossRef]

4) Scarabottolo CC, Cyrino ES, Nakamura PM, et al.: Relationship of different domains of physical activity practice with health-related quality of life among community-dwelling older people: a cross-sectional study. BMJ Open, 2019, 9: e027751. [Medline] [CrossRef]

5) de Vries NM, van Ravensberg CD, Hobbelen JS, et al.: Effects of physical exercise therapy on mobility, physical functioning, physical activity and quality of life in community-dwelling older adults with impaired mobility, physical disability and/or multi-morbidity: a meta-analysis. Ageing Res Rev, 2012, 11: 136-149. [Medline] [CrossRef]

6) Chase JD, Phillips LJ, Brown M: Physical activity intervention effects on physical function among community-dwelling older adults: a systematic review and meta-analysis. J Aging Phys Act, 2017, 25: 149-170. [Medline] [CrossRef]

7) Abdelbasset WK, Alsubaie SF, Tantawy SA, et al.: A cross-sectional study on the correlation between physical activity levels and health-related quality of life in community-dwelling middle-aged and older adults. Medicine (Baltimore), 2019, 98: e14895. [Medline] [CrossRef]

8) Grabara M, Nawrocka A, Powerska-Didkowska A: The relationship between physical activity and work ability—a cross-sectional study of teachers. Int J Occup Med Environ Health, 2018, 31: 1-9. [Medline]

9) López-Bueno R, Andersen LL, Smith L, et al.: Physical activity and perceived stress at work in university workers: a cross-sectional study. J Sports Med Phys Fitness, 2020, 60: 314-319. [Medline] [CrossRef]

10) Robroek SJ, Reeuwijk KG, Hillier FC, et al.: The contribution of overweight, obesity, and lack of physical activity to exit from paid employment: a metaanalysis. Scand J Work Environ Health, 2013, 39: 233-240. [Medline] [CrossRef]

11) Heneweer H, Picavet HS, Staes F, et al.: Physical fitness, rather than self-reported physical activities, is more strongly associated with low back pain: evidence from a working population. Eur Spine J, 2012, 21: 1265-1272. [Medline] [CrossRef]

12) Yabuki S, Fukumori N, Takegami M, et al.: Prevalence of lumbar spinal stenosis, using the diagnostic support tool, and correlated factors in Japan: a population-based study. J Orthop Sci, 2013, 18: 893-900. [Medline] [CrossRef]

13) Kasukawa Y, Miyakoshi N, Kobayashi T, et al.: Limaprost or pregabalin: preoperative and postoperative medication for pain due to lumbar spinal stenosis Pain Pract, 2018, 18: 625-630. [Medline] [CrossRef]

14) Shamji MF, Mroz T, Hsu W, et al.: Management of degenerative lumbar spinal stenosis in the elderly. Neurosurgery, 2015, 77: S68-S74. [Medline] [CrossRef]

15) Gelalis ID, Stafilas KS, Korompilias AV, et al.: Decompressive surgery for degenerative lumbar spinal stenosis: long-term results. Int Orthop, 2006, 30: 59-63. [Medline] [CrossRef]

16) Higuchi D: Influence of chronic pain on perceived health status and physical activity in elderly people after lumbar surgery. Top Geriatr Rehabil, 2018, 34 118-123. [CrossRef]

17) Herno A, Airaksinen O, Saari T, et al.: Pre- and postoperative factors associated with return to work following surgery for lumbar spinal stenosis. Am J Ind Med, 1996, 30: 473-478. [Medline] [CrossRef]

18) Truszczyńska A, Rąpała K, Truszczyński O, et al.: Return to work after spinal stenosis surgery and the patient's quality of life. Int J Occup Med Environ Health, 2013, 26: 394-400. [Medline] [CrossRef]

19) Alghadir AH, Anwer S, Iqbal A, et al.: Test-retest reliability, validity, and minimum detectable change of visual analog, numerical rating, and verbal rating scales for measurement of osteoarthritic knee pain. J Pain Res, 2018, 11: 851-856. [Medline] [CrossRef]

20) Herdman M, Gudex C, Lloyd A, et al.: Development and preliminary testing of the new five-level version of EQ-5D (EQ-5D-5L). Qual Life Res, 2011, 20: 1727-1736. [Medline] [CrossRef]

21) Golicki D, Niewada M, Buczek J, et al.: Validity of EQ-5D-5L in stroke. Qual Life Res, 2015, 24: 845-850. [Medline] [CrossRef]

22) Bilbao A, García-Pérez L, Arenaza JC, et al.: Psychometric properties of the EQ-5D-5L in patients with hip or knee osteoarthritis: reliability, validity and responsiveness. Qual Life Res, 2018, 27: 2897-2908. [Medline] [CrossRef]

23) Shiroiwa T, Ikeda S, Noto S, et al.: Comparison of value set based on DCE and/or TTO data: scoring for EQ-5D-5L health states in Japan. Value Health, 2016, 19: 648-654. [Medline] [CrossRef]

24) Shimizu H: An introduction to the statistical free software HAD: Suggestions to improve teaching, learning and practice data analysis. J Media Inf Commun, 2016, 1: 59-73 (in Japanese with English abstract).

25) Leensen MC, Groeneveld IF, van der Heide I, et al.: Return to work of cancer patients after a multidisciplinary intervention including occupational counselling and physical exercise in cancer patients: a prospective study in the Netherlands. BMJ Open, 2017, 7: e014746. [Medline] [CrossRef]

26) Cauter JV, Bacquer D, Clays E, et al.: Return to work and associations with psychosocial well-being and health-related quality of life in coronary heart disease patients: Results from EUROASPIRE IV. Eur J Prev Cardiol, 2019, 26: 1386-1395. [Medline] [CrossRef] 
27) Murayama H, Amagasa S, Inoue S, et al.: Sekentei and objectively-measured physical activity among older Japanese people: a cross-sectional analysis from the NEIGE study. BMC Public Health, 2019, 19: 1331. [Medline] [CrossRef]

28) Carr LJ, Leonhard C, Tucker S, et al.: Total worker health intervention increases activity of sedentary workers. Am J Prev Med, 2016, 50: 9-17. [Medline] [CrossRef]

29) Gutermuth LK, Hager ER, Pollack Porter K: Using the CDC's worksite health scorecard as a framework to examine worksite health promotion and physical activity. Prev Chronic Dis, 2018, 15: E84. [Medline] [CrossRef]

30) Pulakka A, Leskinen T, Koster A, et al.: Daily physical activity patterns among aging workers: the Finnish Retirement and Aging Study (FIREA). Occup Environ Med, 2019, 76: 33-39. [Medline] [CrossRef]

31) Kehler DS, Clara I, Hiebert B, et al.: Sex-differences in relation to the association between patterns of physical activity and sedentary behavior with frailty. Arch Gerontol Geriatr, 2020, 87: 103972. [Medline] [CrossRef]

32) Olanrewaju O, Kelly S, Cowan A, et al.: Physical activity in community dwelling older people: a systematic review of reviews of interventions and context. PLoS One, 2016, 11: e0168614. [Medline] [CrossRef]

33) Tucker P, Gilliland J: The effect of season and weather on physical activity: a systematic review. Public Health, 2007, 121: 909-922. [Medline] [CrossRef]

34) Yasunaga A, Togo F, Watanabe E, et al.: Sex, age, season, and habitual physical activity of older Japanese: the Nakanojo study. J Aging Phys Act, 2008, 16: 3-13. [Medline] [CrossRef]

35) Mumu SJ, Fahey PP, Ali L, et al.: Seasonal variations in physical activity domains among rural and urban Bangladeshis using a culturally relevant Past Year Physical Activity Questionnaire (PYPAQ). J Environ Public Health, 2019, 2019: 2375474. [Medline] [CrossRef] 